\title{
Dynamic effects of pericardial effusion without tamponade Respiratory responses in the absence of pulsus paradoxus
}

\author{
VICTOR S WAYNE, RICHARD L BISHOP, DAVID H SPODICK \\ From the Cardiology Division, St Vincent Hospital and the University of Massachusetts Medical School, Worcester, \\ Massachusetts, USA
}

SUMMARY Fourteen patients with pericardial effusions without clinical signs of tamponade (pulsus paradoxus or other cardiac and circulatory abnormalities) showed inspiratory reductions in echocardiographic left ventricular internal diastolic diameter which correlated directly with the exaggerated respiratory changes in systolic time intervals (both pre-ejection period and left ventricular ejection time). Excessive pericardial fluid affects left ventricular function even in the absence of clinical manifestations.

Cardiac tamponade interferes with left ventricular function, as indicated clinically by pulsus paradoxus (inspiratory fall in systolic blood pressure $>10 \mathrm{~mm} \mathrm{Hg}$ during quiet breathing). ${ }^{1}$ Recently effusions without clinical signs of tamponade have been shown to have an effect on left ventricular function. ${ }^{2}$ Such effusions are not physiologically inert, as they have respiratory effects on systolic time intervals far in excess of those found in normal subjects ${ }^{3}$ and control subjects with dry pericarditis. ${ }^{2}$ Like pulsus paradoxus, but in its absence, these changes-that is, inspiratory increase in pre-ejection period and decrease in left ventricular ejection time-are exaggerations of normal responses.

Inspiratory changes in systolic time intervals are characteristic of decreased preload ${ }^{4}$ although they also occur with depressed myocardial function and acutely increased afterload. ${ }^{6}$ Since pericardial effusions without tamponade would not justify invasive investigation, we analysed non-invasive data to $(a)$ elucidate the mechanism of changes in systolic time intervals and (b) obtain more information for assessing such patients further. Thus we analysed echocardiographic data in addition to recording systolic time intervals, both measured independently. We considered that if an unbiased study showed correlations between phasic respiratory ventricular dimensions and systolic time intervals, the non-invasive assessment of these patients would be improved and the effects of pericardial effusions without clinical signs of tamponade clarified.

Requests for reprints to: Dr D H Spodick, Cardiology Division, St Vincent Hospital, 25 Winthrop Street, Worcester, Massachusetts 01604, USA.

Accepted for publication 16 September 1983

\section{Patients and methods}

Fourteen patients examined echocardiographically during this study fulfilled the following criteria: a pericardial effusion without echo signs of tamponade ${ }^{7}$; no evidence of pre-existing cardiac or pulmonary disease; good quality echocardiograms; a respiratory variation of $\leqslant 10 \mathrm{~mm} \mathrm{Hg}$ in brachial artery cuff systolic pressure; no jugular venous distension; stable sinus rhythm (electrocardiogram) without notable respiratory changes; and a clear second heart sound on phonocardiograms. Recordings were made on an Irex System II recorder with Leatham suction microphones and filters adjusted for optimal S2.

The following data were recorded at $100 \mathrm{~mm} / \mathrm{s}$ : a left ventricular echocardiogram at chordal level for left ventricular measurement ${ }^{7}$; phasic breathing changes via a thermistor sensor; a phonocardiogram at a position to register clearly the aortic component of S2 (usually mid-left sternal border); and a right carotid displacement pulse. To ensure objectivity, independent observers measured the systolic time intervals and echocardiograms and compared the data only after all measurements had been made.

Systolic time intervals were calculated from electrocardiograms, carotid pulse, and S2 according to standard methods ${ }^{2}$; left ventricular echocardiographic measurements (mean of 6 cycles) included ${ }^{7}(a)$ minimal internal diastolic diameter (inspiration), maximum internal diastolic diameter (expiration), and the difference between the two measurements; $(b)$ fractional shortening and $(c)$ velocity of circumferential fibre shortening. 
During the study a further two patients with noneffusive (dry) pericarditis were identified by a pericardial rub, appropriate electrocardiographic changes, ${ }^{8}$ a syndrome consistent with acute pericarditis, and echocardiograms showing no fluid. The same procedure for measuring systolic time intervals and recording respiratory and echocardiographic data was followed.

\section{Results}

\section{SYSTOLIC TIME INTERVALS}

All 16 patients showed the same directional changes: maximum pre-ejection period and minimum left ventricular ejection time in beats at or closest to peak inspiration. Mean maximal inspiratory pre-ejection period for the 14 patients with effusions was $90.5 \pm 14.77$ (SD) ms; mean change with respiration was $12 \cdot 1 \pm 5 \cdot 45$ (SD) ms. The two patients with dry pericarditis both had a mean change of $5.0 \mathrm{~ms}$. Mean respiratory change in left ventricular ejection time in patients with effusions was $16 \cdot 8 \pm 6.08(\mathrm{SD}) \mathrm{ms}$; the two patients with dry pericarditis both had a mean change of only $5.0 \mathrm{~ms}$.

\section{ECHOCARDIOGRAPHY}

Echocardiographic indices of left ventricular function were: fractional shortening (FS) $=35 \cdot 1 \pm 4.8$ (SD) (normal $36 \cdot 0)^{7}$; velocity of circumferential fibre shortening $(\mathrm{VCF})=1.47 \pm 0.225(\mathrm{SD})$ circumferences/s (normal 1.3 circumferences/s). ${ }^{7}$ Every patient had an inspiratory decrease in left ventricular internal diastolic diameter.

In the Figure changes in left ventricular internal diastolic diameter are plotted against changes in systolic time intervals. Because the respiratory change in each is used, signs (+ or -) are eliminated. In the Figure (a) the change in left ventricular internal diastolic diameter shows an inverse correlation with the change in pre-ejection period $(r=0.76, p<0.01)$ that is, the greater the increase in pre-ejection period with inspiration the greater the reduction in left ventricular internal diastolic diameter with inspiration. The two patients with dry pericarditis had a minimal change in left ventricular internal diastolic diameter $(0.5 \mathrm{~mm}$ and zero), with a correspondingly small change in pre-ejection period of $5.0 \mathrm{~ms}$ in both. The Figure (b) shows the direct correlation $(r=0.84$, $p<0.01$ ) between the changes in left ventricular internal diastolic diameter and left ventricular ejection time. The two patients with dry pericarditis (with 0 and $0.5 \mathrm{~mm}$ changes in left ventricular internal diastolic diameter) each had a correspondingly small change in left ventricular ejection time of $5.0 \mathrm{~ms}$. In general, changes were greater with larger effusions, although attempts to divide effusions into echocardio-
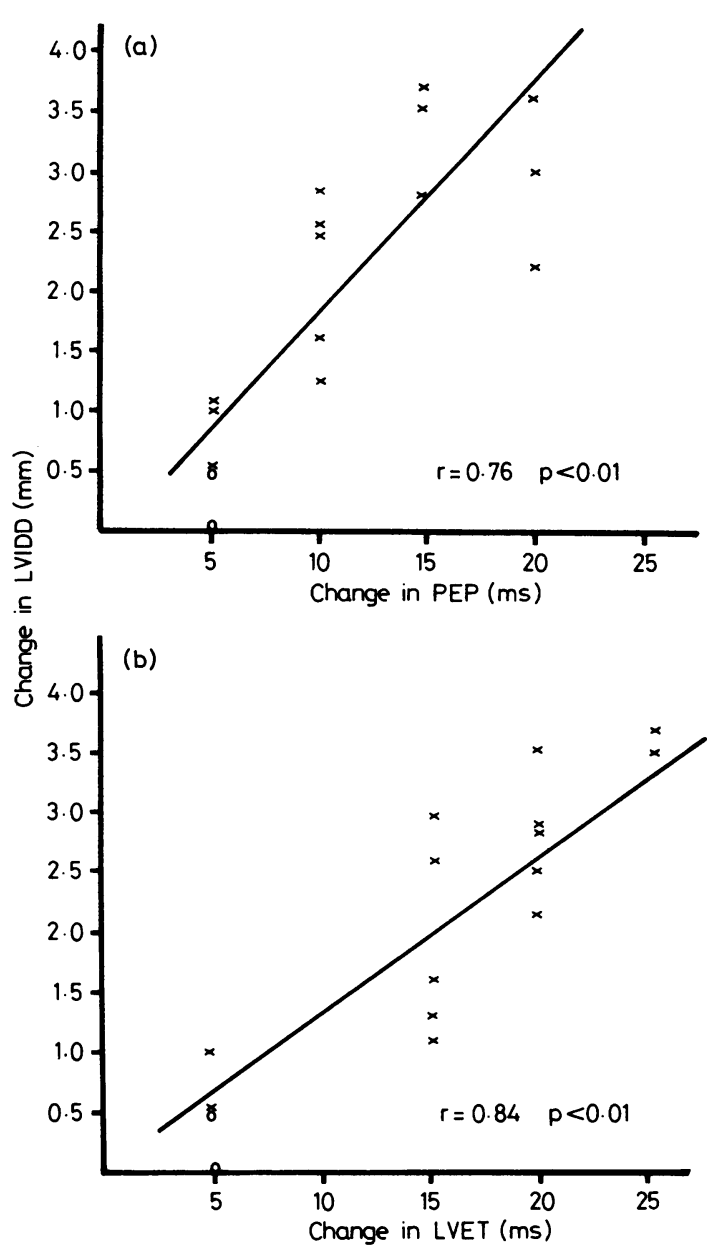

Fig. Relation between the respiratory changes in echocardiographic left ventricular internal diastolic diameter (LVIDD) and (a) in the pre-ejection period (PEP) and (b) in left ventricular ejection time (LVET). In (a) a direction correlation $(r=0.76, p<0.01)$ exists between the changes - that is, with inspiration, the greater the increase in $P E P$, the greater the reduction in LVIDD. In $(b)$ a direct correlation $(r=0.84$, $p<0.01)$ exists - that is, with inspiration the greater the decrease in LVET the greater the reduction in LVIDD. The circles (O) represent the data for the two patients with dry pericarditis; the small changes in LVIDD were associated with correspondingly small changes in LVET.

graphically small, moderate, and large (highly inaccurate at best ${ }^{7}$ ) yielded such small subgroups that tests for statistical differences were not applied.

\section{Discussion}

Respiratory changes in systolic time intervals in normal ambulatory subjects and in patients with stable coronary artery disease are quite small (mean change in 
left ventricular ejection time $7 \cdot 1 \pm 2 \cdot 3(\mathrm{SD}) \mathrm{ms}$; mean change in pre-ejection period $\left.4 \cdot 2 \pm 3 \cdot 3 \mathrm{~ms}^{3}{ }^{9}\right)$. Respiratory changes in systolic time intervals reported for five patients ${ }^{2}$ with dry pericarditis were comparable $(8 \cdot 0 \pm 2 \cdot 73$ and $7 \cdot 0 \pm 2 \cdot 73 \mathrm{~ms}$ for mean changes in left ventricular ejection time and pre-ejection period respectively) and similar to the minor changes in our two patients. In contrast, our patients with pericardial effusions without clinical signs of tamponade showed mean changes of $12 \cdot 1 \pm 5 \cdot 45 \mathrm{~ms}$ and of $16 \cdot 8 \pm 6.08 \mathrm{~ms}$ respectively.

The very good to excellent correlations between respiratory changes in left ventricular internal diastolic diameter and in pre-ejection period and left ventricular ejection time further improve the echocardiographic evaluation of pericardial effusions without clinical signs of tamponade. In keeping with this trend, the two patients with dry pericarditis, in contrast to the group with effusions who had normal changes in systolic time intervals, also showed very small changes in left ventricular internal diastolic diameter. Although changes in left ventricular internal diastolic diameter doubtless represent changes in volume, we have not calculated volume measurements in view of the errors in extrapolating $M$ mode dimensions to volumes.'

Expected respiratory variations in echocardiographic ventricular dimensions reflect inspiratory decrease in left ventricular filling (and consequently preload) from the diminished pulmonary venous return during more negative inspiratory thoracic pressure and encroachment on the left ventricle by right ventricular expansion. ${ }^{10}$ These normal respiratory haemodynamic responses are exaggerated not only in florid cardiac tamponade ${ }^{11}{ }^{12}$ but also, as we have shown, in effusions without clinical signs of tamponade. Our findings differ from some of the results of Settle $e t$ al, who found the same directional respiratory changes in ventricular dimensions during tamponade but were unable to show respiratory changes in a group with effusions without tamponade. ${ }^{12}$ It is surprising that they noted no respiratory variation at all in their patients, in view of the expected normal respiratory variations that occur. ${ }^{70}$ These workers also considered that their findings did not apply in mild tamponade, which contrasts directly with the major implication of our results - that is, that some effusions may represent subclinical tamponade. Moreover, all our patients had the same directional results irrespective of echocardiographic effusion depth. Finally, the blinding procedure gave us confidence in the objectivity of the correlations (Figure).

None of our patients had evidence of cardiac disease. Baseline average pre-ejection period, left ventricular ejection time, and echo measurements of fractional shortening and velocity of circumferential shortening were normal, confirming the clinical conclusion that intrinsic myocardial function was intact and that the changes related to alterations in haemodynamic loading rather than to depressed myocardial function.

That there is some scatter in the results without smooth gradations related to effusion size-even if that could be accurately estimated-is not surprising since one variable, the elasticity of the parietal pericardium, cannot be measured. The latter varies among patients depending on age, present or previous inflammation, and the rate of fluid accumulation ${ }^{13}$ and determines the critical, but individually variable, amount of pericardial fluid required to influence intrapericardial pressure during inspiration and therefore exert a dynamic effect. ${ }^{13}$

The pericardial effusions without clinical signs of tamponade in our patients may represent an intermediate state between normal and overt cardiac tamponade, in which similar mechanisms may cause some degree of cardiac compression, thus indicating that the fluid is not without haemodynamic effect. Such compression may not produce clinical evidence of cardiac tamponade such as pulsus paradoxus, but may be detected by other more sensitive indices including the systolic time intervals and echocardiographic measurements.

\section{References}

1 Shabetai R. The pericardium. New York: Grune \& Stratton, 1981: 79.

2 Spodick DH, Paladino D, Flessas AP. Respiratory effects on systolic time intervals during pericardial effusion. Am $\mathcal{F}$ Cardiol 1983; 51: 1033-5.

3 Pigott VM, Spodick DH. Effects of normal breathing and expiratory apnea on duration of the phases of cardiac systole. Am Heart f 1971; 82: 786-93.

4 Weissler AM, Peeler RG, Roehll WH Jr. Relationships between left ventricular ejection time, stroke volume, and heart rate in normal individuals and patients with cardiovascular disease. Am Heart $\mathcal{F}$ 1961; 62: 367-78.

5 Spodick DH. Effects of dysrhythmias and A-V block on noninvasive evaluation of cardiac status and performance. In: Zoneraich S, ed. Noninvasive methods in cardiology. Springfield: Charles C Thomas, 1974: 362-78.

6 Wallace AG, Mitchell JH, Skinner NS, Sarnoff SJ. Duration of the phases of left ventricular systole. Cir Res 1963; 12: 611-9.

7 Feigenbaum H. Echocardiography. 3rd ed. Philadelphia: Lea \& Febiger, 1981; 478-504, 119-31, 134, 550, 490, 132, 137.

8 Bruce MA, Spodick DH. Atypical electrocardiogram in acute pericarditis: characteristics and prevalence. $f$ Electrocardiol 1980; 13: 61-6.

9 Nandi PS, Pigott VM, Spodick DH. Sequential cardiac responses during the respiratory cycle: patterns of change in systolic intervals. Chest 1973; 63: 380-5.

10 Brenner JI, Waugh RA. Effect of phasic respiration on left ventricular dimension and performance in a normal population. Circulation 1978; 57: 122-7.

11 D'Cruz IA, Cohen HC, Prabhu R, Glick G. Diagnosis of cardiac tamponade by echocardiography. Circulation 1975; 52: 460-5.

12 Settle HP, Adolph RJ, Fowler NO, Engel P, Agruss NS, Levenson NI. Echocardiographic study of cardiac tamponade. Circulation 1977; 56: 951-9.

13 Spodick DH. The normal and diseased pericardium: curren concepts of pericardial physiology, diagnosis and treatment. foumal of the American College of Cardiologists 1983; 1: 240-51. 